\title{
TITLE:
}

\section{$<$ Note> Male Core Areas: Ranging by Budongo Forest Chimpanzees}

$\operatorname{AUTHOR}(\mathrm{S})$ :

Newton-Fisher, Nicholas E.

\section{CITATION:}

Newton-Fisher, Nicholas E.. <Note> Male Core Areas: Ranging by Budongo Forest Chimpanzees. Pan Africa News 2000, 7(1): 10-12

ISSUE DATE:

2000-06

URL:

http://hdl.handle.net/2433/143383

\section{RIGHT:}

Copyright (C) Pan Africa News. 
<NOTE>

Male Core Areas: Ranging by Budongo Forest Chimpanzees

\section{Nicholas E. Newton-Fisher (University of Cambridge)}

Male chimpanzees are generally considered to make fairly even use of their home range, while in contrast, females show more restricted ranging patterns, spending much of their time in small fragments (core areas) of the community's home range. Restricted ranging of (non-cycling) females may relate to either ensuring access to good food resources, or reducing the risk of intercommunity infanticide, while males may range widely to search for potential mates and contest inter-community territorial borders $(1,2)$.

Careful reading of published accounts $(3,4)$ suggests, however, that sex differences in ranging patterns may not be so extreme. In the Gombe National Park, Tanzania, where females have readily identifiable core areas, males are also described as having core areas (3), and in the Kibale National Park, Uganda, males as well as females showed a 'clumped' pattern of range use (4).

Detailed analysis (5) of the ranging patterns of male chimpanzees in the Budongo Forest, Uganda, provides further support for this view. The twelve adult males in this community during 1995 had heterogeneous ranging patterns. Within a small (around $7 \mathrm{~km}^{2}$ ) home range, males occupied non-exclusive, partially overlapping, core areas. Males spent most ( $80 \%)$ of their time within these areas. These core areas existed only in a probabilistic sense, representing 'preferred' areas of activity. Range structure was investigated using both kernel analysis, which 
areas of activity. Range structure was investigated using both kernel analysis, which tends to merge areas, and cluster analysis, which tends to split areas. Kernel analysis identified core areas which averaged $32 \%$ of the community range, [cf. 40-60\% reported for Gombe males (3)], whereas cluster analysis identified multinuclear core areas averaging only $7 \%$ of the community range.

Maintaining core areas through restricted ranging may impose few costs on males. One fifth of the males' time is spent outside these preferred areas, which may be sufficient for the exploitation of ephemeral food sources, and occasional engagement in territorial behaviour (5). It may reduce males ability to search for cycling (particularly peripheral) females, although there is good evidence that females do not remain in their core areas when cycling but leave them to associate with $(6,7,8,9)$ and possibly seek out $(10,11)$ males, which may make male searching less necessary.

Why males should have core areas is a more open question. At a proximate level, males may simply occupy areas which are familiar to them, perhaps because they were parts of their mothers' core areas. Such an hypothesis would be testable with long term data. The risk of attack from males in neighbouring communities may favour avoidance of boundary areas unless and until males are ready for such encounters, but while this would generate a 'core area phenomenon', it would not explain heterogeneity in male ranging patterns. Core areas seem unlikely to function ecologically: Newton-Fisher. (5) found no differences between male core areas in habitat composition, and no influence of social status on the size or degree of overlap of these core areas.

An obvious product of spending most of the time within a restricted area is that location becomes relatively predictable. As the size of the restricted area decreases, predictability should increase. Could having a predictable location be advantageous for male chimpanzees, and so provide possible social function for core areas? In a mixed forest such as Budongo, attenuation of sound is likely to be unpredictable, with the result that 'pant hoot' vocalisations could reliably convey information about direction, but not distance. If preferred areas of activity lay in the direction indicated by a vocalisation, this would give any listener a probable location for the caller. The advantage to the male within his core area may be the more rapid response of allies. During 1995 the Sonso community saw a change in alpha male, and some shuffling of social status between males, and it might be that the tension associated with instability in the hierarchy, and the need for allies to locate each other, led to greater geographical separation of the adult males and the appearance of defined core areas. Other habitats may impose different limitations on the usefulness of calls as a means of locating individuals and so favour core areas.

The expression of male core areas may be conditional upon a particular community's habitat and demography. The adult sex ratio, in particular, may have important consequences. If the ratio is highly skewed with many adult females, the benefits (probability of future paternity) associated with searching for cycling peripheral females may exceed those gained by being locatable. This may result in heterogeneous patterns of range use, but perhaps not in. obviously distinguishable core areas. In Budongo the adult sex ratio during 1995 was close to $1: 1$ (12), and males may not have benefited by investing much time in searching for cycling females. However, the evidence from Gombe and Kibale (Kanyawara) of male core areas comes from communities with female skewed sex ratios. Perhaps the benefits associated with searching for peripheral females are generally too low to warrant much of a male's time.

It may be that particular patterns of food abundance and dispersion may be necessary for males to show this highly heterogeneous pattern of ranging. The particular pattern of food abundance and distribution in Budongo may have allowed males to spend much time in only a small fraction of their range. Equally abundant but more tightly clumped food would potentially increase the homogeneity in ranging patterns and so limit the expression of differentiated core areas by increasing the amount of time community members visited the same locations on the same days. A habitat with particularly low 
levels of food abundance may place heavy demands on foraging, with resultant extensive ranging and an increase in homogeneity between male ranging patterns. The larger core areas, both relatively and absolutely, seen at Gombe may be the result of overall lower levels of food abundance, although could equally be linked to the female-skewed sex ratio.

A closer look at male ranging patterns seems warranted, as they may reveal a more complex picture of chimpanzee sociality and behavioural diversity.

\section{REFERENCE}

(1) Wrangham, R.W. 1986. Ecology and social relationships in two species of chimpanzee. In: Ecology and Social Evolution: Birds and mammals. Rubenstein, D.I. \& Wrangham, R.W. (eds.), Princeton University Press, Princeton, USA, pp. 352-378.

(2) Dunbar, R.I. 1988. Primate Social Systems. Croom Helm, London. 373 pages.

(3) Wrangham, R.W. \& Smuts, B.B. 1980. Sex differences in the behavioural ecology of chimpanzees in the Gombe National Park, Tanzania. Journal of Reproduction and Fertility, Supplement, 28: 13-31.

(4) Chapman, C.A. \& Wrangham, R.W. 1993. Range use of the forest chimpanzees of Kibale: Implications for the understanding of chimpanzee social organization. Am. J. Primatol., 31: 263-273.

(5) Newton-Fisher, N.E. in press. Ranging patterns of male chimpanzees in the Budongo Forest, Uganda: range structure and individual differences. In: $\mathrm{NeW}$ perspectives in Primate Evolution and Ecology. Harcourt, C. (ed.), Westbury Publishing.

(6) Tutin, C.E.G. 1979. Mating patterns and reproductive strategies in a community of wild chimpanzees (Pan troglodytes schweinfurthii). Behav. Ecol. Sociobiol., 6: 29-38.
(7) Nishida, T. 1979. The social structure of chimpanzees of the Mahale Mountains. In: The Great Apes. Hamburg, D.A. \& McCown: E.R. (eds.), Benjamin/Cummings Publishing Company, Menlo Park, CA; pp. 73-122.

(8) Goodall, J. 1986. The Chimpanzees of Gombe: Patterns of behaviour. Belknap Press, Cambridge, MA. 673 pages.

(9) Nishida, T. \& Hiraiwa-Hasegawa, M. 1987. Chimpanzees and bonobos: Cooperative relationships between males. In: Primate Societies. Smuts, B.B., Cheney, D.L., Seyfarth, R.M., Wrangham, R.W., \& Struhsaker, T.T. (eds.), University of Chicago Press, Chicago, pp. 165-177.

(10) Gagneux, P., Woodruff, D.S. \& Boesch, C. 1997. Furtive mating in female chimpanzees. Nature, 387: 358-359.

(11) Takahata, Y., Ihobe, H. \& Idani, G. 1996 Comparing copulations of chimpanzees and bonobos: Do females exhibit proceptivity or receptivity? In: Great Ape Societies. McGrew, W.C., Marchant, L.F. \& Nishida, T. (eds.), Cambridge University Press, Cambridge, pp. 146-155.

(12) Newton-Fisher, N.E. 1997. Tactical behaviour and decision making in wild chimpanzees. PhD. University of Cambridge. 